\title{
Wirelessly Powered Embedded Sensor Nodes for Internal Structural Health Monitoring
}

\author{
Zhihua Wang and Andrew Markham
}

\begin{abstract}
Monitoring large civil structures during construction and over their entire lifetime is key to predicting structural weaknesses and providing predictive maintenance. Existing approaches to structural health monitoring typically rely on wired or wireless sensors placed on the exterior of the structure. These approaches, though easy to power and replace and provide useful information, fall short of measuring what is happening deep within the structure itself. Wireless sensors embedded inside are particularly attractive for this type of applications, but its lifetime is limited by battery capacity. Charging these sensors has been the bottleneck. Existing wireless power transfer approaches based on electromagnetic propagation suffer heavy attenuation due to the high dielectric absorption of the dense concrete. To resolve the issue, the limitation of dielectric absorption is tackled with a novel physical layer. In this work, power transfer is built on the fact that nearly all concrete structures are reinforced with steel bars, also known as rebar, to provide tensile strength. Instead of transferring power over a few centimetres of concrete with a large transmitter, the reinforcing steel can act as a conduit for extremely low frequency $(<200 \mathrm{~Hz})$ magneto-inductive fields. To exploit the energy conduction via the magnetic conduit, an equivalent circuit based on physical model of multi-coil coupling over rebar is derived and mathematically analysed. Practical experiments demonstrate that the transmission distance of rebar-rebar coupled wireless power transfer is an order of magnitude further than airconcrete coupled wireless power transfer, allowing sensors to be realistically powered at distances approaching $700 \mathrm{~mm}$.
\end{abstract}

Index Terms-Magnetic resonant coupling, wireless power transfer, wireless sensor networks.

\section{INTRODUCTION}

$\mathbf{E}$ NERGY harvesting and wireless power transfer (WPT) have become a heated topic as a result of challenges in powering mobile and sensor devices. Within the umbrella of WPT, the three most widely known mechanisms are inductive coupling [1], [2], strongly coupled magnetic resonance [3]-[5] and far-field radio frequency harvesting using a rectenna [6], [7]. In recent research, WPT has been explored as a sustainable energy supply for wireless sensor networks (WSN) [8], [9], and structural health monitoring (SHM) [10], [11]. Longterm structural health monitoring, in particular, is the key

Manuscript received Dec 17, 2019; revised Apr 06, 2020; accepted Jul 03, 2020.

This work was supported by Engineering and Physical Sciences Research Council (EPSRC EP/M017583/1)

The authors are with University of Oxford, Oxford, United Kingdom (zhihua.wang@cs.ox.ac.uk, andrew.markham@cs.ox.ac.uk) to providing information about the safety and performance of large civil structures (e.g., bridges and buildings). This work will thus focus on challenges of acquiring sensor data from within the structure itself, i.e. embedded within concrete beams, pillars and foundations.

Wired sensors are extensively used but complex to install and connections are prone to break in case of structural damage. More critically, the wire ingress point can accelerate water seepage, increasing corrosion of the steel reinforcing [12], [13]. Wireless sensors, on the other hand, bear numerous advantages in terms of deployment flexibility and operational robustness. However, it is clear that for long-term deployments of concrete-embedded sensors, primary batteries are not a viable option. The majority of WSN-based power transferring techniques target operation in free-space, i.e. transmission of power through air. However, WPT through obstacles (e.g., concrete, soil and pipeline) has been underinvestigated. Penetration, reflection and attenuation losses can be very challenging for such power transmissions. Charging embedded sensors through obstacles is more critical in these applications due to the impossibility of replacing batteries without damaging the structure. This is particularly important for critical civil infrastructures that typically demand longterm condition monitoring over decades. To this end, recent work has demonstrated the feasibility of powering a sensor at a depth of $9 \mathrm{~cm}$ within reinforced concrete using a $900 \mathrm{MHz}$ carrier, with an efficiency of $1.32 \%$ [14]. Downhole telemetry using modulated reflectance was investigated for oil-well monitoring [15], [16], which indicated the possibility of using a pipeline as a waveguide. In general, magnetic resonance based WPT within concrete can achieve a range of several millimetres to centimetres [17]. The short transmission range results from high moisture content in concrete that leads to significant electromagnetic absorption, as well as the rebar mesh structure inside concrete that causes Faraday cage shielding. Alternative approaches like piezoelectric energy harvesting [18] have been applied to SHM to supply microwatt levels of power, but this depends on sufficient vibrations of the structure, which is not always the case (e.g., foundations or piles).

This research proposes a new WPT method for wireless sensors nodes embedded deep inside concrete. Unlike previous work attempting to avoid the rebar's lossy impact, the rebar is exploited as an efficient conduit for very- and extremely- low frequency magnetic fields $(<200 \mathrm{~Hz})$. Both circuit theory and numerical simulations of rebar channel are analysed to explain how low frequency magnetic fields propagate through a steel rod. Although rebar cage, rebar net, and single rebar have 


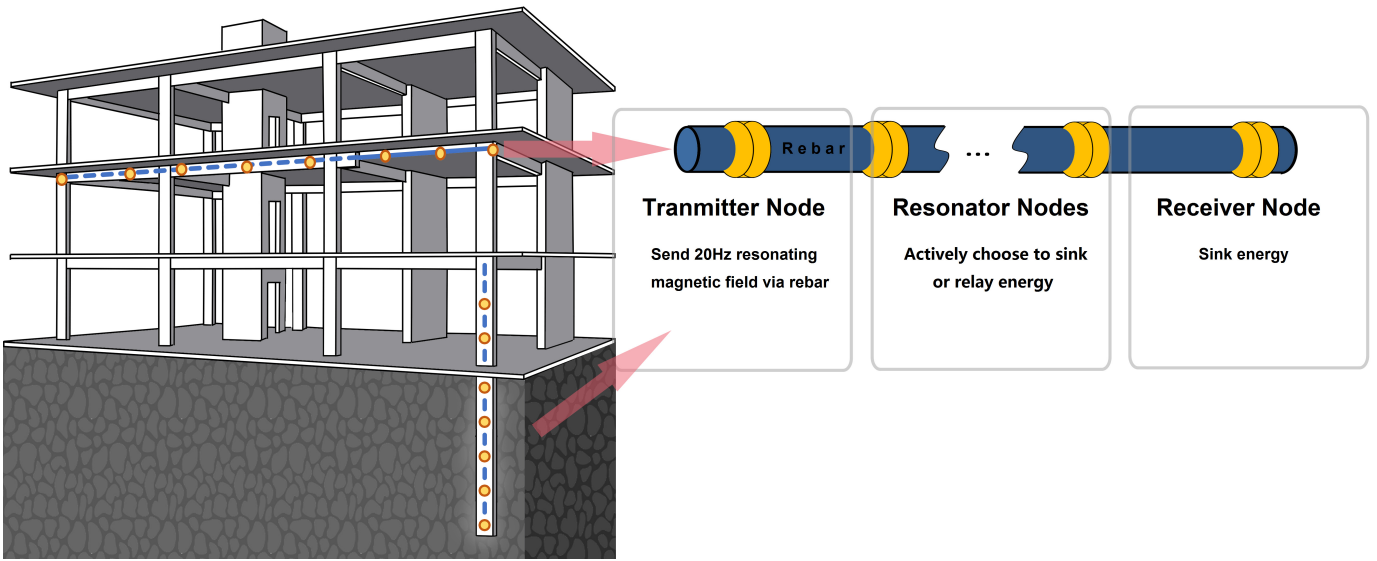

Fig. 1. Conceptual model of the proposed system.

different geometric structures, this paper primarily studies the physics phenomena of a single rebar. Based on the theoretical and numerical model, the key factors are discussed to optimise WPT, allowing around 1 Watt to be delivered at a range of $0.1 \mathrm{~m}$, and several $\mathrm{mW}$ to be harvested up to $0.7 \mathrm{~m}$ from a $10 \mathrm{~W}$ transmitter, whilst using miniature 25 -mm-diameter coils. The transmission distance is approximately an order of magnitude deeper than prior work [14] and opens up the possibility of new wireless sensing applications. This work further presents a system design and demonstrates that the experimental and theoretical results coincide in laboratory tests.

In particular, the contributions of this research work are:

- A novel analytical model describing the coupling through the rebar, which employs a novel inductive constantphase-element.

- Validation of the model through extensive simulations and practical experiments.

- Demonstrations of miniature coils' power transfer over long distance through the rebar core, sufficient to charge an embedded low-power sensor.

The paper is organised as follows: Both the circuit and physics of rebar coupled WPT in theory are presented in Section II. The equivalent circuit representing rebar guided WPT is also derived. In section III, the rebar guided WPT is designed, fabricated and measured systematically. The measured results are further compared with the analytical and simulated results.

\section{The Rebar-WPT Principle}

\section{A. System Overview}

The transmitters and receivers are formed by coils wrapped around the steel reinforcing in a rebar-rebar coupled WPT system, as the high-level conceptualisation of proposed system shown in Fig. 1. Sensors embedded within concrete beams and foundations can be wirelessly powered by exploiting existing reinforcing steel. Intermediate resonator nodes can boost the effective coupling to more distant receivers. The transmitter can be externally powered (e.g., through a solar panel) for

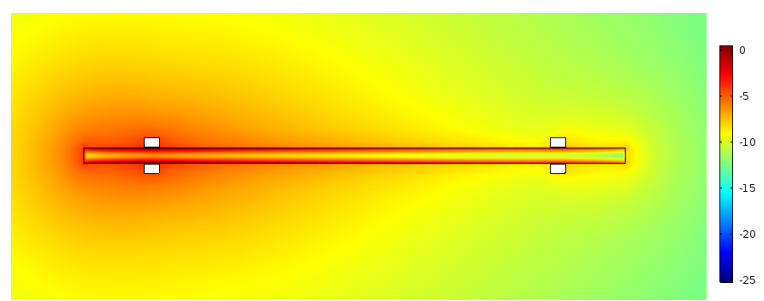

(a)

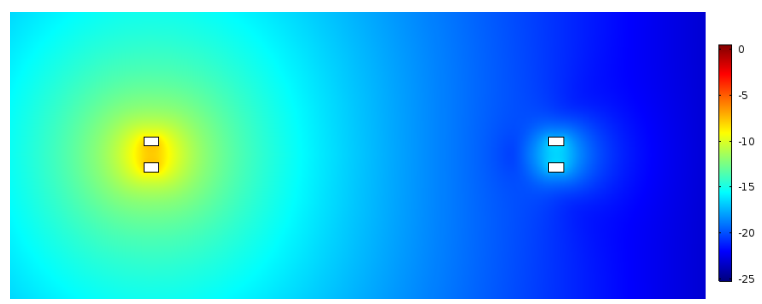

(b)

Fig. 2. Simulated magnetic flux density from the transmitter (left coil) to the receiver (right coil) in cross-sectional view. (a) Rebar Channel (b) Air Channel / Logarithmic Tesla in Base 10.

continuous monitoring in critical applications (e.g., foundations and beams). It can also be employed as intermittent nondestructive testing (NDT) after significant events like an earthquake or bridge strike, where the building inspector can place a transmitter at the test point and query embedded sensors. To relay energy to end nodes more efficiently, the intermediate resonators are also analysed. The rebar itself acts as a core, and as such, behaves like a conduit for the magnetic flux. The nodes and the rebar itself are encased in concrete.

The magnetic flux density is simulated with a nominal $1 \mathrm{~W}$ power supply for both air-channel and rebar-channel. Both are tuned at best efficiency after optimisation. As Fig. 2 (a) shows, the rebar functions as a magnetic conduit, concentrating the flux density linearly along the steel core. In contrast, in Fig. 2 (b) the flux density in air close to the coil is very high, but rapidly vanishes in the near field due to the exponential decay as well as loose coupling. Unlike WPT through air, the rebar-rebar WPT channel has 
several complex physical factors coupled with each other. The complex transmission mechanism is disentangled into separate physical elements, explaining the properties of each element, and eventually formulating an analytical equivalent circuit of the rebar channel.

\section{B. Physical Elements}

1) Rebar Material Properties: Steel reinforcing is specified by structural engineers according to the needs of the structure and the predicted loads. Rebar can range in diameters from $20 \mathrm{~mm}$ to $50 \mathrm{~mm}$ and comes in various grades, corresponding to different strengths and costs. The most widely employed type, in buildings and non-critical infrastructure, is low-carbon steel, also known as mild steel. Due to its ubiquity, mild steel is used throughout this research, but steels of other grades are also discussed for completeness. Table I lists the electric and magnetic properties of most commonly used steels in the construction industry. Compared to air and concrete with an approximate permeability of 1 , the permeability of mild steel can reach up to 280 [19], which concentrates the magnetic flux along the material. As an initial approximation, this increases the coupling between coils by over two orders of magnitude relative to air. However, as discussed below, other factors act to reduce this coupling.

For high tensile strength or corrosive applications, stainless steels are used. Ferritic stainless steel and Martensitic stainless steel can perform even better for WPT due to higher permeability and resistivity. However, there is a special case in Austenitic stainless steel, which is non-magnetic, as the proposed WPT method only works effectively with magnetic materials. This is due to the heat treatment in the metallurgical casting process. Note that even for conventional magnetic SHM method like Magnetic particle inspection (MPI), Austenitic stainless steel can not be tested [20]. In this case, this proposed method does not consider non-magnetic materials.

2) Eddy Currents: An externally time-varying magnetic field induces a perpendicular current flow in a conductor, termed an eddy current. This current, in turn, induces a secondary magnetic field that opposes the primary magnetic field, partially cancelling it out. As a result, the magnetic flux density is large near the surface of the conductor, and decreases inside the conductor. This undesired effect increases overall magnetic reluctance, which can be prominent as frequency increases.

On the assumption that applied materials are homogeneous, power losses $W$ caused by eddy currents are given by [21].

$$
W=\frac{\pi^{2}}{6} \frac{\sigma d^{2} J^{2} f}{\delta}
$$

The related parameters can be found to determine the loss, $\sigma$ is the electrical conductivity, $d$ is the thickness of the magnetic conductor, $J$ is the magnetic polarisation, $f$ is the frequency and $\delta$ is the density of the material.
TABLE I

MAGNETIC AND ELECTRICAL PROPERTIES OF STEEL MATERIALS [19], [22].

\begin{tabular}{ccc}
\hline $\begin{array}{c}\text { Type of } \\
\text { Steel }\end{array}$ & $\begin{array}{c}\text { Relative } \\
\text { Permeability }\end{array}$ & $\begin{array}{c}\text { Resistivity } \\
(\mu \Omega \cdot \mathrm{cm})\end{array}$ \\
\hline Low-Carbon Steel & $100-280$ & 18 \\
Ferritic Stainless Steel & $1000-2600$ & 60 \\
Martensitic Stainless Steel (annealed) & $750-950$ & 55 \\
Martensitic Stainless Steel (hardened) & $40-95$ & 55 \\
Austenitic Stainless Steel & $1-7$ & $72-90$ \\
\hline
\end{tabular}

3) Hysteresis Loss, Quality Factor and Operation Frequency: When the coil is conducting current in one direction, it generates magnetic fields that magnetise the rebar material. This magnetisation is left behind within the rebar when the imposed current is removed, and is referred to as the remanent magnetisation. By applying a field in the opposite direction, the remanent magnetisation is removed with an associated energy loss (as heat), namely hysteresis loss. The Hysteresis effect indicates that low operating frequency will be more efficient due to lower remanent magnetisation energy losses, as shown in the formula below,

$$
P_{b}=\eta B_{\max }^{n} f
$$

where $P_{b}$ is the hysteresis loss per unit volume, $\eta$ is Steinmetz hysteresis coefficient depending on material, $B_{\max }$ is maximum flux density, $n$ is Steinmetz exponent, ranging from 1.5 to 2.5 depending on material, and $f$ is the frequency of magnetic reversals per second.

The quality factor ( $\mathrm{Q}$ factor) describes the resonance behaviour of the coupled coil power transfer system. A higher $\mathrm{Q}$ factor allows the transmitter and receiver to resonate with greater amplitudes. An ordinary WPT can be simplified as a series circuit with a resistor, an inductor, and a capacitor,

$$
Q=\frac{1}{R} \sqrt{\frac{L}{C}}=\frac{\omega L}{R}
$$

where $R$ is equivalent resistance, $L$ is equivalent inductance, which includes mutual inductance and self-inductance, $C$ is additional capacitance, and $\omega$ is angular frequency. The $\mathrm{Q}$ factor is frequency dependent and a high $\mathrm{Q}$ factor is easier to achieve with a higher resonating frequency.

Clearly, there must be an optimal operating frequency that balances the increase in energy transfer with high Q-factor and hysteresis loss. As shown in experimental results in the following sections, the operating frequency is critical for transmission efficiency.

4) Coil Geometry: The rebar forms a path for conducting the magnetic field. The winding height and length, and air gap between the rebar and coil result in another path for the magnetic flux to take. In the case, when a coil has a considerable winding height, the second path needs to be taken into consideration. The air path has a higher $\mathrm{Q}$ and transmits power more efficiently at higher frequencies without hysteresis and eddy current losses. However, the coupling of the air path decays significantly as the transmission distance increases. On the contrary, the rebar path has 
higher permeability than air, which conducts the magnetic field better over longer distances. To conclude, a long, flat solenoid transmits power more efficiently over long ranges. Conversely, a thick, short coil with many deep windings performs better over short ranges. This trade-off between coil size and transmission range can be optimised depending on application constraints.

5) Variable Self-inductance: Generally, magnetic cores can be classified into two categories, namely open core and closed core. The rebar is considered as an open core with a large air gap. Based on magnetic circuit analysis, the magnetic reluctance surrounding the air gap is larger than the reluctance in other parts along the rebar. The magnetic flux distribution near the gap is altered due to reluctance variance. As a coil moves axially along the rebar, its magnetic flux through the rebar is reduced, leading to a drop in self-inductance.

\section{Circuit Theory}

As mentioned previously, a simple WPT model based on coupled air-core coils [23] is shown in Fig. 3 (a). The basic circuit model of a coil consists of an inductor and a resistor in series connection. The inductance encapsulates self-inductance and mutual-inductance, where the mutual-inductance is a result of other coupling with adjacent coils. Both DC and AC resistance contribute to the overall resistance. This WPT model typically operates in the high frequency band (tens of $\mathrm{MHz}$ ), where AC resistance can be considerably large.

Although equivalent circuits have been extensively researched for WPT during the past decade, most are limited to air-core transmitter coils and receiver coils. However, the design and optimisation of power transfer can be very different depending on the core and intermediate medium. For instance, transferring power through tissue for biomedical capsule endoscope [24] is a challenging research area. RamRakhyani et.al, Jow and Ghovanloo [25], [26] derived a more comprehensive circuit model that takes into account the biological tissue as the transmission medium. This can be applied in biomedical applications to charge implanted or encapsulated devices. As shown in Fig. 3 (b), the model introduces a parallel parasitic capacitance due to tissue effects.

In our system, the coil winding around ferromagnetic rebar introduces a dependent coupling between the air path and rebar path, rendering existing circuit theory unable to describe the power transmission mechanism. In this research, a new equivalent circuit model is derived by abstracting the multicoupled physical effects in both paths.

The magnetic flux surrounding a coil in a simple WPT model is shown in Fig. 4 (a). The rebar core alters the flux pattern, as shown in Fig. 4 (b), concentrating the flux nonlinearly along the axis of the core. This effectively creates two magnetic flux paths: one through the air and another along with the rebar. Both paths are represented in detail in the analytical model. The equivalent circuit is shown in Fig. 3 (c). The overall current in a coil consists of an induced current $i_{u}$ via a non-ferromagnetic path (e.g., air and concrete), an effective current $i_{e}$ via ferromagnetic path (e.g., rebar) as well

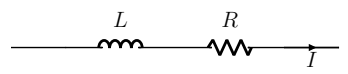

(a)

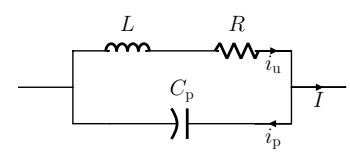

(b)

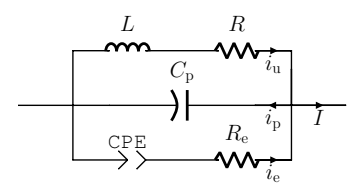

(c)

Fig. 3. Coil equivalent circuit for (a) Air WPT (b) WPT through biomedical tissues (c) Rebar WPT.

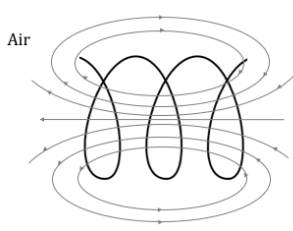

(a)

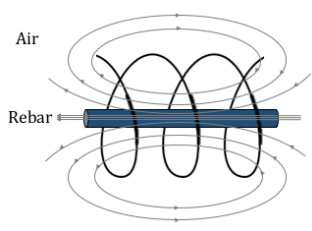

(b)
Fig. 4. Magnetic flux lines around a current carrying coil (a) for air core (b) for rebar core.

as parasitic current $i_{p}$ due to the high dielectric constant of the concrete. While the induced current and effective current increase the achievable energy transmission distance, the parasitic current decreases the energy. Like the simple WPT model, the mechanism of non-ferromagnetic path remains the same. However, the ferromagnetic path works in a different way, which requires a novel formulation.

To correctly model the coupling in circuit theory through the ferromagnetic path, a constant phase element (CPE) is introduced to best represent the complex multi-coupled physics mechanism, extending the prior model [27]. A CPE is widely used to represent the imperfection of real-world capacitors and batteries due to electrochemical effects such as the existence of ionic charge carriers [28] or blocked diffusion in electrodes [29]. The effect of a CPE is to add a constant phase offset to a Nyquist plot, effectively compressing the radius of the magnitude-phase semicircle. The CPE parameter is often determined or fitted in electrochemical impedance spectroscopy (EIS) from a real capacitor's impedance response. In addition, a constant phase effect has been previously used to model steel's electrical properties in the time domain [30], [31]. To the best of our knowledge, this is the first work that has demonstrated how it can accurately represent magnetic properties in WPT and is validated by experimental evidence.

A CPE has two elements, the inductive element and the resistive element. Since the CPE's phase does not alter with frequency, its resistive component increases rapidly along with inductance as frequency increases. On one hand, the undesired resistance of ferromagnetic path can lead to higher losses. On 


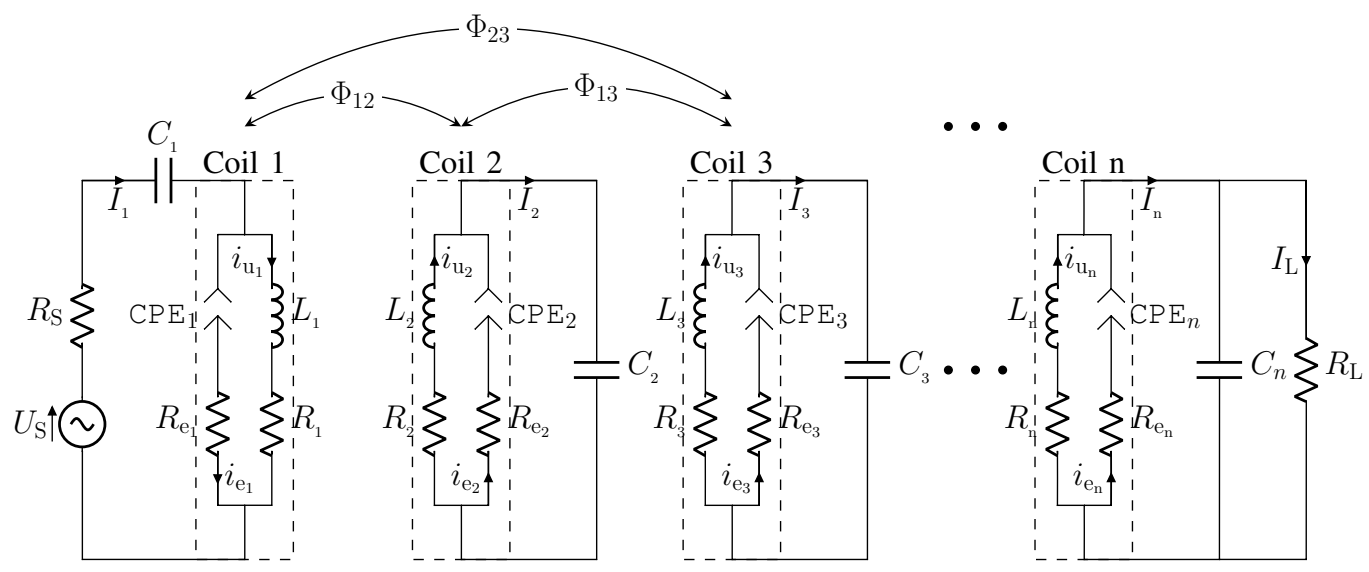

Fig. 5. Equivalent circuit for Rebar-guided WPT.

the other hand, it also has a stronger mutual coupling link between multiple coils over longer distances. The high loss and high coupling are regarded as a contradictory trade-off which needs to be optimised. It is worth to point out that despite the losses in the rebar path due to the resistance in $\mathrm{CPE}$ component, it has a much higher coupling compared to air, especially over long distances.

To integrate a completed WPT system, a matched capacitor $C$ tunes the circuit into resonance. As the transmission operates at very low frequencies $(<200 \mathrm{~Hz})$, this capacitor needs to be relatively large. To simplify the circuit, the parasitic capacitance is neglected. The circuit model is composed for multiple coils, including a transmitter, a receiver and multiple resonators as shown in Fig. 5. The effective coupling is represented as $\Phi_{\mathrm{ij}}$ which is a measure of the mutual linkage between the $i t h$ coil and the $j t h$ coil,

$$
\Phi_{\mathrm{ij}}=A e^{-p x}-B e^{-q x},
$$

where $A, B, p, q$ are derived empirically from experiments/simulations and $x$ is the distance between the coils. Define the load voltage at receiver is $V_{\mathrm{L}}$ equals to $R_{\mathrm{L}} I_{\mathrm{L}} \cdot n$ is the number of coils.

Apply Kirchhoff's law in the transmitter,

$$
V_{\text {coil }_{1}}=V_{\mathrm{S}}-\left(R_{\mathrm{S}}+X_{\mathrm{C}_{1}}\right) I_{1}
$$

where, $V_{\text {coil }_{1}}$ is the output voltage across the coil, $X_{\mathrm{C}_{1}}$ is the reactance of the additional capacitance, $V_{S}$ and $R_{S}$ are the voltage and inner resistance of the AC source, respectively. Based on coils's equivalent circuit for Rebar WPT in Fig. 3 (c), the equation (5) can be written as,

$$
V_{\mathrm{coil}_{1}}=i_{\mathrm{u}_{1}} Z_{\mathrm{U}_{1}}+\sum_{i=2}^{n} \Phi_{1 \mathrm{i}} i_{\mathrm{u}_{\mathrm{i}}}
$$

$$
Z_{\mathrm{U}_{1}}=R_{1}+X_{\mathrm{U}_{1}}
$$

where $Z_{\mathrm{U}_{1}}$ is the overall impedance, $R_{1}$ accounts for ohmic losses of winding (i.e. copper losses), $X_{\mathrm{U}_{1}}$ represents the equivalent reactance of coil, $i_{\mathrm{u}_{\mathrm{i}}}$ is induced current flow in the ith coil, $\Phi_{1 \mathrm{i}}$ is mutual coupling between transmitter and the ith coil. For the ferromagnetic path,

$$
\begin{gathered}
V_{\mathrm{coil}_{1}}=i_{\mathrm{e}_{1}} Z_{\mathrm{E}_{1}} \\
Z_{\mathrm{E}_{1}}=R_{\mathrm{e}_{1}}+C P E_{1}
\end{gathered}
$$

where $Z_{\mathrm{E}_{1}}$ is the overall impedance in the ferromagnetic path, $R_{\mathrm{e}_{1}}$ is the parallel resistance, $C P E_{1}$ is the equivalent impedance due to magnetic conduction and resistance in rebar core.

Based on (4) - (9), the voltage at the source can be obtained:

$$
\begin{array}{r}
i_{\mathrm{u}_{1}}\left[Z_{\mathrm{U}_{1}}+\frac{\left(R_{\mathrm{S}}+X_{\mathrm{C}_{1}}\right)\left(Z_{\mathrm{E}_{1}}+Z_{\mathrm{U}_{1}}\right)}{Z_{\mathrm{E}_{1}}}\right]+ \\
\sum_{i=1}^{n} i_{\mathrm{u}_{\mathrm{i}}} \Phi_{1 \mathrm{i}}\left[\frac{R_{\mathrm{S}}+X_{\mathrm{C}_{1}}+Z_{\mathrm{E}_{1}}}{Z_{\mathrm{E}_{1}}}\right]=V_{\mathrm{S}}
\end{array}
$$

Similarly, the $j$ th resonator can be derived as

$$
i_{\mathrm{u}_{\mathrm{j}}}\left[Z_{\mathrm{U}_{\mathrm{j}}}+\frac{X_{\mathrm{C}_{\mathrm{j}}}\left(Z_{\mathrm{E}_{\mathrm{j}}}+Z_{\mathrm{U}_{\mathrm{j}}}\right)}{Z_{\mathrm{E}_{\mathrm{j}}}}\right]-\sum_{i=1}^{n} i_{\mathrm{u}_{\mathrm{i}}} \Phi_{\mathrm{ji}}\left(\frac{X_{\mathrm{C}_{\mathrm{j}}}+Z_{\mathrm{E}_{\mathrm{j}}}}{Z_{\mathrm{E}_{\mathrm{j}}}}\right)=0
$$

Therefore, the matrix of this model can be derived as (12). By characterising a rebar-core coil through impedance analysis or numerical simulation, the matrix can be used to determine the system efficiency.
$\left[\begin{array}{cccc}Z_{U_{1}}+\left(R_{\mathrm{S}}+X_{C_{1}}\right)\left(\frac{Z_{\mathrm{U}_{1}}}{Z_{\mathrm{E}_{1}}}+1\right) & \Phi_{12}\left(\frac{R_{\mathrm{S}}+X_{\mathrm{C}_{1}}}{Z_{\mathrm{E}_{1}}}+1\right) & \Phi_{13}\left(\frac{R_{\mathrm{S}}+X_{\mathrm{C}_{1}}}{Z_{\mathrm{E}_{1}}}+1\right) & \cdots \\ -\Phi_{21}\left(\frac{X_{\mathrm{C}_{2}}}{Z_{\mathrm{E}_{2}}}+1\right) & Z_{\mathrm{U}_{2}}+X_{\mathrm{C}_{2}}\left(\frac{Z_{\mathrm{U}_{2}}}{Z_{\mathrm{E}_{2}}}+1\right) & -\Phi_{23}\left(\frac{X_{\mathrm{C}_{2}}}{Z_{\mathrm{E}_{2}}}+1\right) & \cdots \\ \vdots & \vdots & \vdots & \\ -\Phi_{(\mathrm{n}-1) 1}\left(\frac{X_{\mathrm{C}_{\mathrm{n}-1}}}{Z_{\mathrm{E}_{\mathrm{n}}-1}}+1\right) & \cdots & \cdots & \cdots \\ -\Phi_{\mathrm{n} 1}\left(\frac{X_{\mathrm{C}_{\mathrm{n}}} R_{\mathrm{L}}}{Z_{\mathrm{E}_{\mathrm{n}}}\left(X_{\mathrm{C}_{\mathrm{n}}}+R_{\mathrm{L}}\right)}+1\right) & \cdots & \cdots & \cdots\end{array}\right.$

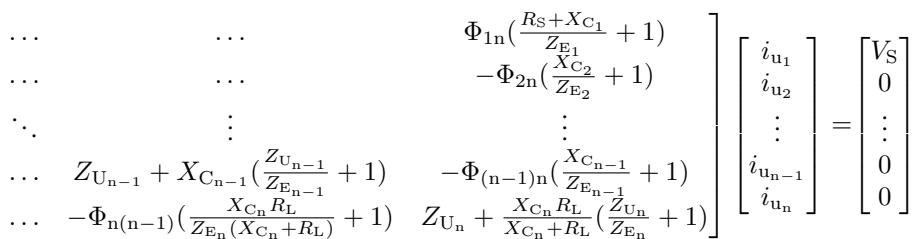




\section{DESIGN, IMPLEMENTATION AND EXPERIMENTAL VERIFICATION OF THE WPT SYSTEM}

\section{A. System Setup}

Our aim is to design a system that can couple the maximum flux into the rebar and then extract the maximum energy out of it. This is considered to be an optimisation problem with several variables, including operating frequency, resonant tuning capacitance, wire thickness and number of turns. The constrains of the system and numerical computations are imposed as follows:

1) Dimension: The rebar used in these experiments has a length of $800 \mathrm{~mm}$ and diameter of $20 \mathrm{~mm}$. The inner diameter of the solenoid coil is set to be $20 \mathrm{~mm}$. The outer diameter and length are limited to $35 \mathrm{~mm}$ and $25 \mathrm{~mm}$, respectively. The limitation on size is to satisfy deployment requirements in practical construction process. For simplicity, all coils are made identical, although a heterogeneous arrangement for transmitters, resonators and receivers could yield efficiency improvements. Note that considering the size and diameter of coils, it is extremely challenging for WPT to transfer power over hundreds of millimetres, i.e. over a hundred diameters.

2) Load Impedance: An energy harvester designed for high voltage spikes (e.g., piezoelectric harvester) has a high input impedance. In contrast, a $10 \Omega$ small load impedance is applied to match our coil model and power conversion circuit.

3) Power Supply: A normal charging power of $10 \mathrm{~W}$ source is used at the transmitter. This is easily achievable in structural health monitoring applications with a small, external solar panel. This can easily be increased to tens or even hundreds of watts depending on the application.

4) Numerical Setup: In addition to the analytical model, a numerical model is further established based on finite element simulation software (COMSOL). Finite element analysis discretises the physical space and then solves boundary value problems between adjacent cells. Non-linear ferromagnetic materials is introduced in simulation. The geometric properties for numerical setup is the same as those used for experimental setup, and material properties in COMSOL analysis were initially based on the parameters listed in Table 1 but optimised through empirical studies.

5) Computational Setup: In terms of the computing time, each run takes approximately three minutes on a workstation (Intel Xeon CPU E5-1603 v3 @ 2.80G x 4). In light of the dimensionality of the search space, the model is excuted on a 1024-core computer cluster ( 8 nodes x 16 CPUs x 8 cores), which can compute the same programme in approximately $100 \mathrm{~ms}$ for each run. Therefore, for the numerical model the transmission efficiency can be optimised over multiple dimensions (e.g., frequency, distance, number of turns, gauge, capacitance).

\section{B. System Implementation}

Fig. 6 illustrates the characterisation of rebar-core coil by using a Keysight E5061B Vector Network Analyser (VNA). The VNA has a frequency range from $5 \mathrm{~Hz}$ to $500 \mathrm{MHz}$. The coil's leads are connected to VNA through test fixture. By analysing the impedance, the effective coupling parameters $\Phi$

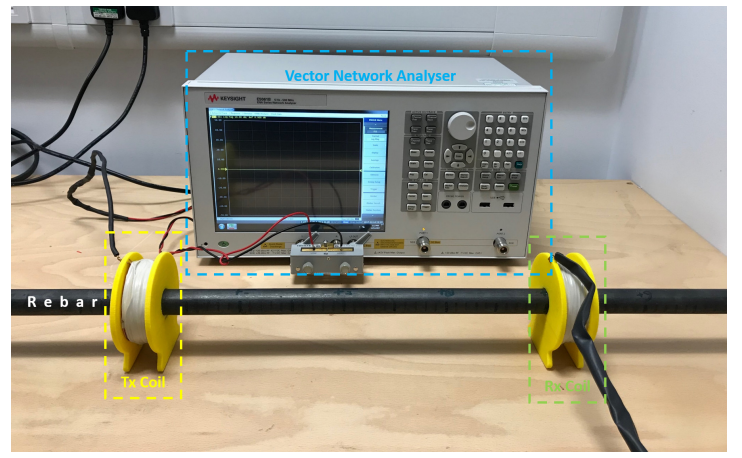

Fig. 6. Configuration for rebar-core coil characterisation.

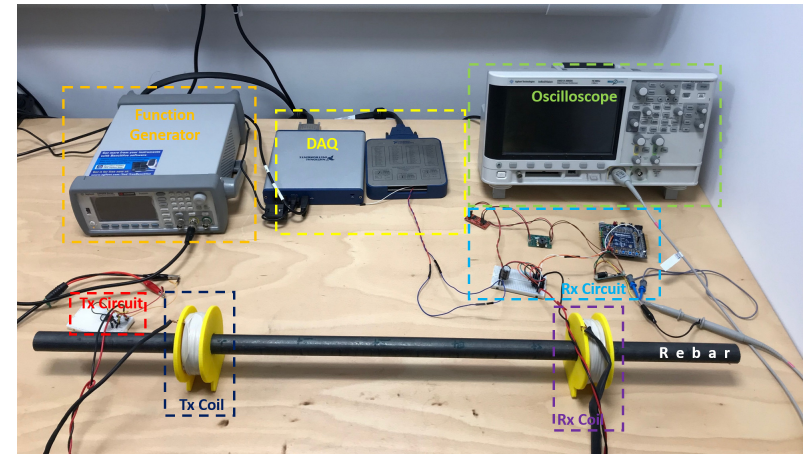

Fig. 7. Test environment for power transmission through rebar.

can be derived. The test environment of the rebar-WPT system is shown in Fig. 7.

A major challenge is harvesting power from low voltage sources. For example, assuming a coil power of $1 \mathrm{~W}$ is applied at $20 \mathrm{~Hz}$, the voltage at a distance of $700 \mathrm{~mm}$ away from the transmitter is only $14 \mathrm{mV}$ RMS (impedance matched for optimal efficiency). From Faraday's law, the low rate of change of flux linkage with time makes the induced voltage small. Therefore, a cascaded circuit is designed to draw energy from milivolts, which includes a Charge Pump, a Voltage Booster and a Storage Recifier as shown in Fig. 8.

The charge pump is an 8-stage voltage multiplier, which consists of MOSFET arrays. Ultra low supply voltage of $10 \mathrm{mV}$ and nanowatt low power operation Advanced Linear Devices ALD210800 MOSFET is employed in these experiments. An optional step-up transformer with ratio 1:10 can be connected following the charge pump to further increase voltage level. The voltage booster can output the voltage to $6 \mathrm{~V}$ starting from $2 \mu \mathrm{W}$ and $60 \mathrm{mV}$. The Storage Rectifier will store the energy in a $0.1 \mathrm{~F}$ supercapacitor. When sufficiently charged, the power good (PGOOD) signal is used to power-up the circuitry. The rectifier provides a stable $3.3 \mathrm{~V}$ supply to microcontroller. The Ambiq Apollo microcontroller employed has quiescent current of nanoamps and works at $35 \mu \mathrm{A} / \mathrm{MHz}$.

ABS coil formers were 3D printed. 400 turns of $1 \mathrm{~mm}$ diameter solid core copper wire were wound into the form stacking into 20 layers. The coil was thus around $25 \mathrm{~mm}$ wide by $15 \mathrm{~mm}$ high in cross-section. Tolerances between coils were less than $0.5 \%$, tested by an impedance analyser. The data acquisition unit NI 7856R DAQ was used to collect data. The 


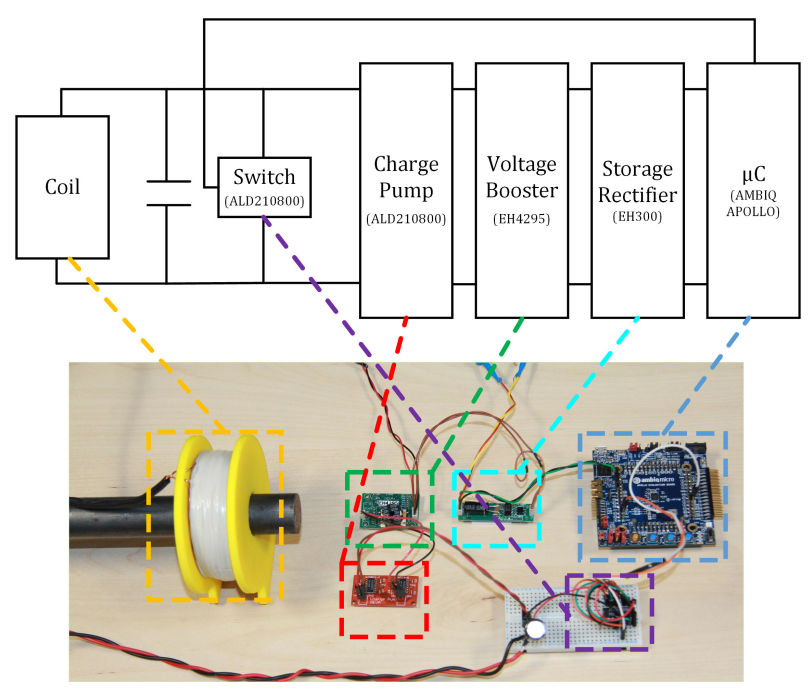

Fig. 8. Block diagram and photograph of our prototype node, showing main components.

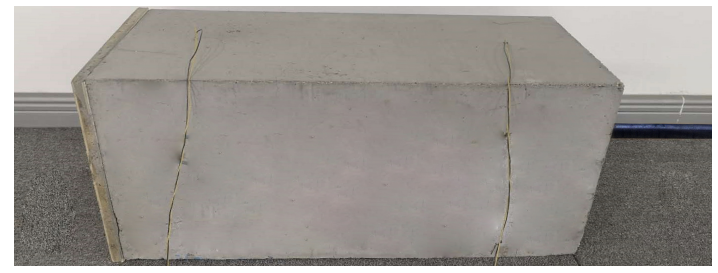

Fig. 9. Validation of power transmission along an $800 \mathrm{~mm}$ rebar with a transmitter and a receiver cast in concrete.

rebar and coils were cast into concrete in the final experiment to thoroughly validate the predictions, as shown in Fig. 9. However, due to the large number of test points involved for data collection and the difficulty of setting up experiment solely with concrete, this experiment uses test results derived from a combination of concrete test points and sand test points.

\section{Experimental Results}

A close correspondence between the numerical, analytical and experimental characterisation of a single coil coupled to the rebar-core are shown on a Bode Plot in Fig. 10. Both the magnitude and phase plots illustrate a strong correspondence between the numerical and analytical results. The experimental results are in strong correspondence over $100 \mathrm{~Hz}$. Below $100 \mathrm{~Hz}$, the errors are likely to be a result of the VNA as it worked near its frequency limit. The result highlights the importance of the inclusion of the CPE in the analytical model, without which the phase error would be significantly higher. This justifies the use of the CPE, a component which has not been used before in a WPT model.

Given a 10 Watt power supply, the harvested energy are analysed in numerical, analytical and experimental results in Fig. 11. Fig. 11 (a) shows the power harvested at receiver against distance along a rebar at $20 \mathrm{~Hz}$ and $80 \mathrm{~Hz}$ respectively, with a $10 \mathrm{~W}$ input power. The received power of WPT measured across a $10 \Omega$ resistor. The analytical results are based on the circuit theory model derived in Sec. II with parameters

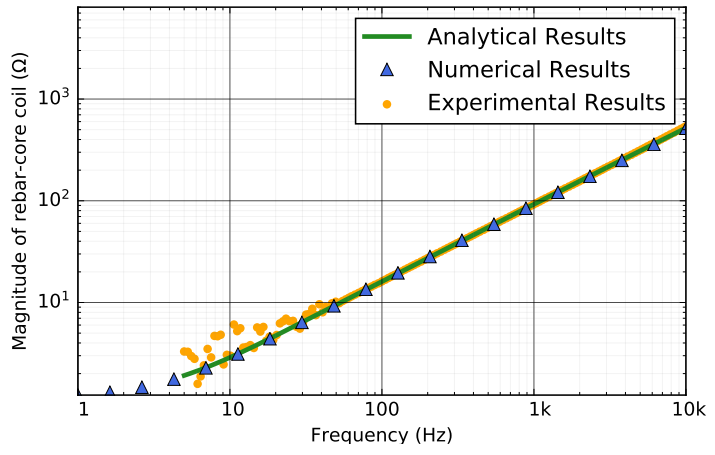

(a)

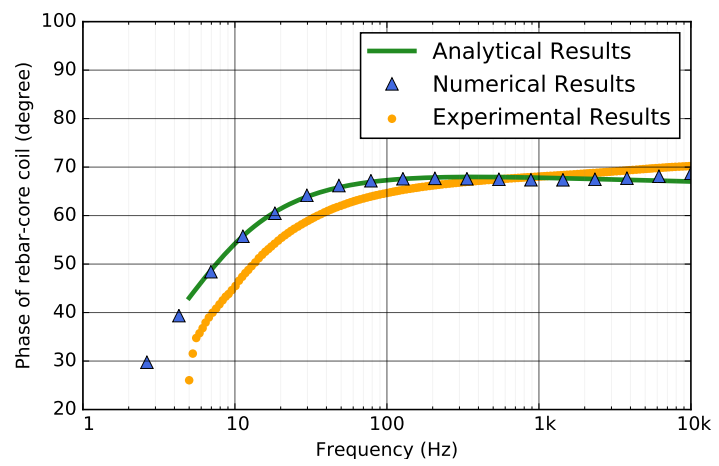

(b)

Fig. 10. Bode plot of rebar-core coil in numerical simulation, exp erimental results and derived circuit model (a) in magnitude; (b) in phase.

fitted from the numerical model, as shown in Table II. These results further demonstrate that the simulated, analytical and experimental results are in very close correspondence, showing that the underlying physics of the rebar channel have been accurately modelled. At a transmission frequency of $20 \mathrm{~Hz}$, it can be seen that $10 \mathrm{~mW}$ can be harvested at $400 \mathrm{~mm}$ and $300 \mu \mathrm{W}$ at $700 \mathrm{~mm}$.

The harvested power with variation in operating frequency is shown in Fig. 11 (b). As a baseline, the results of aircoupled coils are also included, which are tuned to resonate with same dimensions, operating at $13.56 \mathrm{MHz}$ (e.g., using the QI standard [32]). Although the air-coupled WPT at short distances leads to higher efficiency, it rapidly falls off beyond a 3-coil diameter's transmission distance, with a $60 \mathrm{~dB} / \mathrm{dec}$ ade loss. For the rebar-coupled WPT model, it can be seen that at higher frequencies (e.g., $80 \mathrm{~Hz}$ ), efficiency is high at short distances but the slope of the rolloff is higher than at lower frequencies (e.g., $20 \mathrm{~Hz}$ ). Note the coil dimensions are the same, both air-channel and rebar-channel are optimised at best efficiency. The system can deliver around $300 \mu \mathrm{W}$ at a distance of $700 \mathrm{~mm}$ under $20 \mathrm{~Hz}$. It is relatively simple at these frequencies to inject tens or even hundreds of watts with an amplifier to increase the amount of power that can be harvested or to extend the overall distance.

The operating frequencies were swept from $5 \mathrm{~Hz}$ to $200 \mathrm{~Hz}$ with 5 fixed transmission distances. Fig. 11 (c) shows the optimal frequency drops as the increases of transmission distance. 


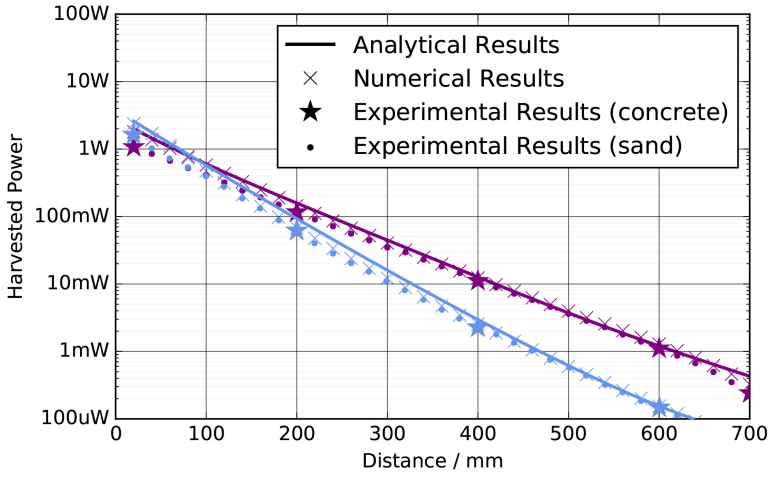

(a)

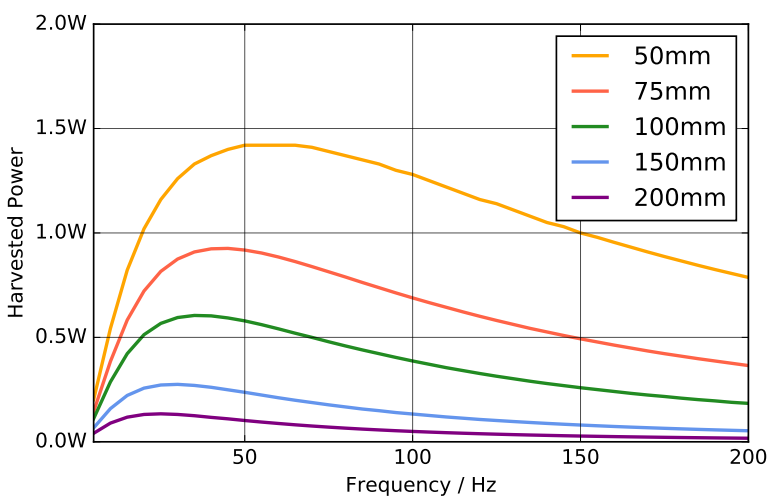

(c)

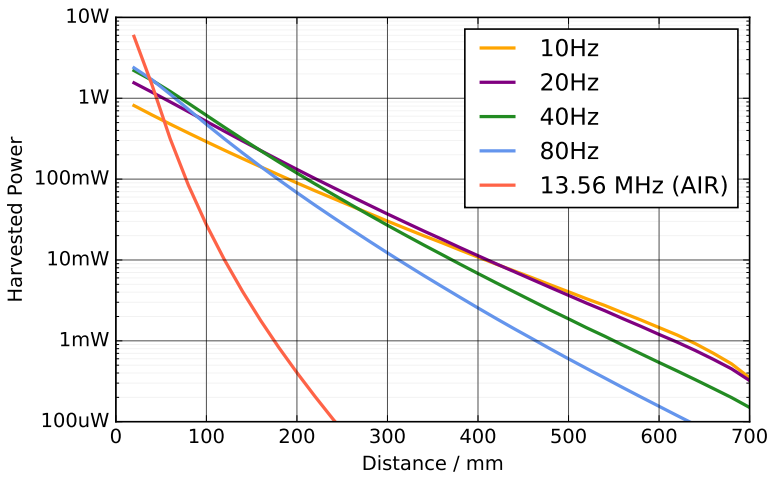

(b)

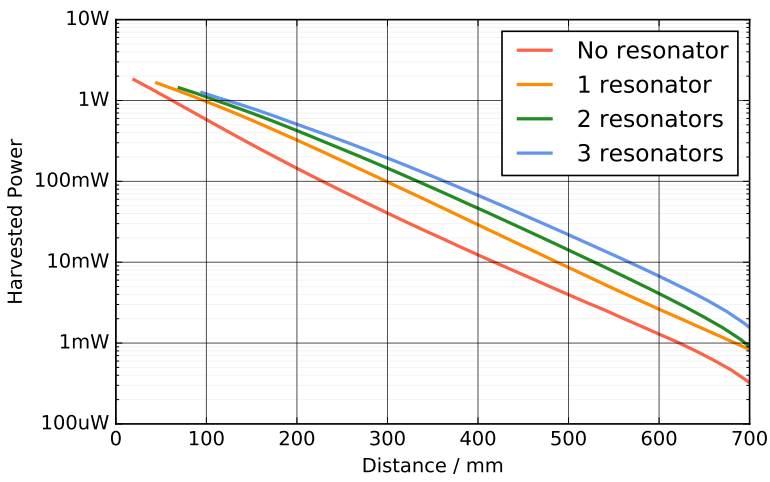

(d)

Fig. 11. Harvested power results (a): at receiver against distance along a rebar at $20 \mathrm{~Hz}$ (purple) and $80 \mathrm{~Hz}$ (blue); (b): versus distance at various frequencies; (c): versus frequencies at various distances, (d): are enhanced by using intermediate unloaded resonators. Harvested power results in (b) (c) (d) are produced from numerical results.

TABLE II

Rebar Core Coil Parameters.

\begin{tabular}{ccccc}
\hline $\mathrm{L}(\mathrm{mH})$ & $\mathrm{R}(\Omega)$ & $\mathrm{Rp}(\Omega)$ & $\mathrm{CPE}-\mathrm{T}(S)$ & CPE-P \\
\hline 121.3 & 4.01 & 1.246 & 5.62 & -0.73 \\
\hline \hline $\mathrm{A}$ & $\mathrm{B}$ & $\mathrm{p}$ & $\mathrm{q}$ & \\
\hline 26.67 & 14.55 & $6.04 \mathrm{e}-3$ & $10.7 \mathrm{e}-3$ & \\
\hline
\end{tabular}

This is caused by self-inductance variance and change in the dominant path i.e. the ferromagnetic or non-ferromagnetic path (through concrete, rather than along the rebar). For a shorter transmission distance of $50 \mathrm{~mm}$, a high power of around $1.5 \mathrm{~W}$ was obtained at relatively high frequency at around $50 \mathrm{~Hz}$. However, for a longer transmission distance of $0.2 \mathrm{~m}$, a peak power of $100 \mathrm{~mW}$ was harvested at lower frequency $25 \mathrm{~Hz}$. This further validates that in the case of short distance, the non-ferromagnetic path allows resonance at a higher frequency with higher power harvested as illustrated in the previous section.

Power transfer can be further improved by introducing intermediate resonators as shown in Fig. 11 (d), acting as a lumped transmission line. The harvested power is compared from zero to three resonators over various distances. The resonators are spaced evenly between transmitter and receiver.
Note that resonators can switch roles and act as receivers easily by just turning on or off the their load circuitry (e.g., a sensor in this case). In the case of three resonators, the amount of power is increased from around $500 \mathrm{~mW}$ to $1 \mathrm{~W}$ at $0.1 \mathrm{~m}$, and improved from around $300 \mu \mathrm{W}$ to around $2 \mathrm{~mW}$ at $0.7 \mathrm{~m}$. This result demonstrates that useful amounts of power can be harvested at significant distances by employing intermediate sensors along the rebar which can act as resonators.

\section{CONCLUSION}

In this paper, the reinforcing steel within concrete structures was studied as a conduit for very low frequency magnetic fields. Multiple coupled physics effects were disentangled behind this simple idea of power transmission though rebar. Based on these physics effects, a new circuit model was introduced, which brought the CPE to the WPT family, in order to model the power transmission mechanism of coils along the rebar core. The close correspondence between analytical, numerical and experimental results indicated that $\mathrm{CPE}$ represented the model accurately. In addition, the dual-path effects were further analysed including ferromagnetic path and non-ferromagnetic path, as well as each one's impact on transmission performance. Power transmission was optimised in a finite element model in high dimension search space, including operating frequencies, tuning capacitors, number of turns, 
gauges, working distances etc. Intermediate resonators were also introduced to boost power efficiency. A receiver circuit was established, which could harvest micro-watt level power from milli-volts voltage. The results were further validated from experiments even with a piece of cast concrete. This paper serves as a first step to creating sustainable energy supply for embeddable structural health sensors. The ferromagnetic channel can be further developed as a communication channel to drive integrated wireless power and communication in future work.

\section{REFERENCES}

[1] C. Carretero, "Coupling power losses in inductive power transfer systems with litz-wire coils," IEEE Transactions on Industrial Electronics, vol. 64, no. 6, pp. 4474-4482, 2017.

[2] F. van der Pijl, P. Bauer, and M. Castilla, "Control method for wireless inductive energy transfer systems with relatively large air gap," IEEE Transactions on Industrial Electronics, vol. 60, no. 1, pp. 382-390, 2013.

[3] Z. Zhang, K. Chau, C. Liu, F. Li, and T. Ching, "Quantitative analysis of mutual inductance for optimal wireless power transfer via magnetic resonant coupling," IEEE Transactions on Magnetics, vol. 50, no. 11, pp. 1-4, 2014.

[4] H. Hu and S. V. Georgakopoulos, "Multiband and broadband wireless power transfer systems using the conformal strongly coupled magnetic resonance method,' IEEE Transactions on Industrial Electronics, vol. 64, no. 5, pp. 3595-3607, 2017.

[5] J. Shin, S. Shin, Y. Kim, S. Ahn, S. Lee, G. Jung, S.-J. Jeon, and D.-H. Cho, "Design and implementation of shaped magnetic-resonance-based wireless power transfer system for roadway-powered moving electric vehicles," IEEE Transactions on Industrial Electronics, vol. 61, no. 3, pp. 1179-1192, 2014.

[6] S. Kim, R. Vyas, J. Bito, K. Niotaki, A. Collado, A. Georgiadis, and M. M. Tentzeris, "Ambient rf energy-harvesting technologies for selfsustainable standalone wireless sensor platforms," Proc. IEEE, vol. 102, no. 11 , pp. 1649-1666, 2014.

[7] A. Collado, S.-N. Daskalakis, K. Niotaki, R. Martinez, F. Bolos, and A. Georgiadis, "Rectifier design challenges for rf wireless power transfer and energy harvesting systems," Radioengineering, vol. 26, no. 2, p. 411, 2017.

[8] N. B. Carvalho, A. Georgiadis, A. Costanzo, H. Rogier, A. Collado, J. A. Garcia, S. Lucyszyn, P. Mezzanotte, J. Kracek, D. Masotti et al., "Wireless power transmission: R\&d activities within europe," IEEE Transactions on Microwave Theory and Techniques, vol. 62, no. 4, pp. 1031-1045, 2014

[9] T. Hosseinimehr and A. Tabesh, "Magnetic field energy harvesting from ac lines for powering wireless sensor nodes in smart grids," IEEE Transactions on Industrial Electronics, vol. 63, no. 8, pp. 4947-4954, 2016.

[10] X. Wang and A. Mortazawi, "Medium wave energy scavenging for wireless structural health monitoring sensors," IEEE Transactions on Microwave Theory and Techniques, vol. 62, no. 4, pp. 1067-1073, 2014.

[11] Y. Lee, D. Blaauw, and D. Sylvester, "Ultralow power circuit design for wireless sensor nodes for structural health monitoring," Proc. IEEE, vol. 104, no. 8, pp. 1529-1546, 2016.

[12] Y. Yu, G. Qiao, and J. Ou, "Self-powered wireless corrosion monitoring sensors and networks," IEEE Sensors Journal, vol. 10, no. 12, pp. 19011902,2010

[13] K. Perveen, G. E. Bridges, S. Bhadra, and D. J. Thomson, "Corrosion potential sensor for remote monitoring of civil structure based on printed circuit board sensor," IEEE transactions on instrumentation and measurement, vol. 63, no. 10, pp. 2422-2431, 2014.

[14] S. Jiang and S. V. Georgakopoulos, "Optimum wireless powering of sensors embedded in concrete," IEEE Transactions on Antennas and Propagation, vol. 60, no. 2, pp. 1106-1113, 2012.

[15] S. D. Briles, D. L. Neagley, D. M. Coates, and S. M. Freund, "Remote down-hole well telemetry," Jul. 20 2004, uS Patent 6,766,141.

[16] D. M. Coates, M. C. Thompson, D. W. Beck, M. E. Gonzalez, M. A. Kendrick, and D. L. Neagley, "Downhole sensor interrogation employing coaxial cable," Jan. 17 2017, uS Patent 9,547,104.

[17] J.-M. Kim, M. Han, and H. Sohn, "Magnetic resonance-based wireless power transmission through concrete structures," J. Electromagn. Eng. Sci., vol. 15, no. 2, pp. 104-110, 2015.
[18] H. Xia, Y. Xia, Y. Ye, L. Qian, and G. Shi, "Simultaneous wireless strain sensing and energy harvesting from multiple piezo-patches for structural health monitoring applications," IEEE Transactions on Industrial Electronics, 2018.

[19] N. Bowler, "Frequency-dependence of relative permeability in steel," in AIP Conference Proceedings, vol. 820, no. B, p. 1269. IOP Institute of Physics Publishing Ltd, 2006.

[20] M. Lovejoy, Magnetic particle inspection: a practical guide. Springer Science \& Business Media, 2012.

[21] F. Fiorillo, Characterization and measurement of magnetic materials. Academic Press, 2004.

[22] J. R. Davis et al., Stainless steels. ASM international, 1994

[23] S. Y. R. Hui, W. Zhong, and C. K. Lee, "A critical review of recent progress in mid-range wireless power transfer," IEEE Transactions on Power Electronics, vol. 29, no. 9, pp. 4500-4511, 2014.

[24] M. R. Basar, M. Y. Ahmad, J. Cho, and F. Ibrahim, "An improved wearable resonant wireless power transfer system for biomedical capsule endoscope," IEEE Transactions on Industrial Electronics, vol. 65, no. 10, pp. 7772-7781, 2018

[25] A. K. RamRakhyani, S. Mirabbasi, and M. Chiao, "Design and optimization of resonance-based efficient wireless power delivery systems for biomedical implants," IEEE Transactions on Biomedical Circuits and Systems, vol. 5, no. 1, pp. 48-63, 2011.

[26] U.-M. Jow and M. Ghovanloo, "Modeling and optimization of printed spiral coils in air, saline, and muscle tissue environments," IEEE transactions on biomedical circuits and systems, vol. 3, no. 5, pp. 339347, 2009.

[27] Z. Wang, O. Kypris, and A. Markham, "Repwr: Wireless power transfer within reinforced concrete," in Proceedings of the 4th International Workshop on Energy Harvesting and Energy-Neutral Sensing Systems, pp. 1-6. ACM, 2016.

[28] M. S. Abouzari, F. Berkemeier, G. Schmitz, and D. Wilmer, "On the physical interpretation of constant phase elements," Solid State Ionics, vol. 180, no. 14, pp. 922-927, 2009.

[29] J. Bisquert, G. Garcia-Belmonte, P. Bueno, E. Longo, and L. Bulhoes, "Impedance of constant phase element (cpe)-blocked diffusion in film electrodes," Journal of Electroanalytical Chemistry, vol. 452, no. 2, pp. 229-234, 1998.

[30] V. Feliu, J. González, C. Andrade, and S. Feliu, "Equivalent circuit for modelling the steel-concrete interface. i. experimental evidence and theoretical predictions," Corrosion Science, vol. 40, no. 6, pp. 975-993, 1998.

[31] A. Sagüés, S. Kranc, and E. Moreno, "The time-domain response of a corroding system with constant phase angle interfacial component: application to steel in concrete," Corrosion Science, vol. 37, no. 7, pp. 1097-1113, 1995.

[32] D. Van Wageningen and T. Staring, "The qi wireless power standard," in Power Electronics and Motion Control Conference (EPE/PEMC), 2010 14th International, pp. S15-25. IEEE, 2010.

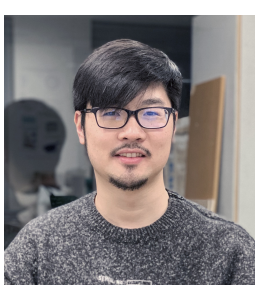

Zhihua Wang is a DPhil student at the Department of Computer Science, University of Oxford. Before that, he obtained MPhil degree from University of Cambridge and BEng degree from University of Manchester and North China Electric Power University. His research interests lie in Cyber Physical systems, deep learning and intuitive physics. He is currently working on multi-modality time-series prediction on sensor data and applications in energy and healthcare sectors.

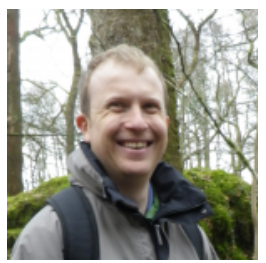

Andrew Markham is an associate professor in the Department of Computer Science, at the University of Oxford. He received his BSc (Hons)(2004) degree and $\mathrm{PhD}(2008)$ degree both from the University of Cape Town, South Africa. He broadly works within the area of cyberphysical systems and machine learning, with application to a number of interdisciplinary areas such as wildlife tracking, earthquake monitoring and industrial safety. Current research interests include data driven techniques for positioning, scene reconstruction and sensor processing. 